Published as:

Nardi T., Canal L., Hausmann M., Dujonc F., Michaud V., Månson J.-A.E., Leterrier Y., Stress Reduction Mechanisms during Photopolymerization of Functionally Graded Polymer Nanocomposite Coatings, Prog. Org. Coat., 87, 204-212 (2015). DOI 10.1016/j.porgcoat.2015.06.004 (C) 2015. This manuscript version is made available under the CC-BY-NC-ND 4.0 license http://creativecommons.org/licenses/by-nc-nd/4.0/

\title{
Stress reduction mechanisms during photopolymerization of functionally graded polymer nanocomposite coatings
}

\section{Nardi Tommaso, Luis Canal†, Michael Hausmann, Florian Dujonc, Véronique Michaud, Jan-Anders Månson, Yves Leterrier*}

\section{Laboratoire de Technologie des Composites et Polymères (LTC) \\ Ecole Polytechnique Fédérale de Lausanne (EPFL) \\ CH-1015 Lausanne, Switzerland}

\author{
* Corresponding Author (yves.leterrier@epfl.ch) \\ $\dagger$ Present Address: \\ Laboratory of Applied Mechanics and Reliability Analysis (LMAF) \\ École Polytechnique Fédérale de Lausanne (EPFL) \\ Station 9, CH-1015 Lausanne, Switzerland
}

\begin{abstract}
From the experimental analysis of the photocuring process in terms of reaction kinetics as well as modulus and shrinkage build-up, the residual stresses arising during the photopolymerization of functionally graded composite coatings based on an acrylate matrix and $\mathrm{Fe}_{3} \mathrm{O}_{4} @ \mathrm{SiO}_{2}$ core@shell nanoparticles are evaluated through a Finite Element Modeling approach. Owing to the monotonous variation of volume fraction of the constituent phases that influences the local conversion of the polymeric matrix, these coatings are able to decrease the residual stresses at the coating/substrate interface by as much as $\approx 25 \%$ compared to those encountered in composites with homogeneous compositions, and by as much as $\approx 40 \%$ compared to those arising in the pure polymer. The influence of substrate stiffness, nanoparticle stiffness and conversion degree of the polymer matrix was also analyzed, providing further information for the optimization of the stress reduction mechanism in graded nanocomposite coatings.
\end{abstract}

\section{KEY WORDS}

Functionally graded materials; residual stress; nanocomposites; photopolymerization; magnetic nanoparticles. 


\section{INTRODUCTION}

In the last years, the study of many bio-composites has elucidated how their mechanical performance tightly relates to the hierarchical organization of their constitutive elements. These elements operate at different levels and in a synergistic fashion, forming complex and non-uniform macroscopic architectures that perform extremely well under the loading conditions to which they are mostly subjected [1-4]. Beyond doubt, bio-composites take full advantage not only of the intrinsic properties of the single components, but also of their spatial distribution in the global structure. The application of this concept to the design of artificial materials would most probably solve many of the problems currently arising both at the manufacturing stage and during lifetime.

It has already been largely shown how properly designed metal-ceramic functionally graded coatings, which provide an artificial approach to the smooth interfacial transitions of biocomposites, can eliminate the stress discontinuity encountered in classical laminated materials by providing a monotonous variation of volume fraction of constituent phases [5-7]. On the contrary, less attention has been paid to functionally graded polymer-based materials [8], even though they could find applications in many technologies currently using thin layers of dissimilar materials in order to achieve functional requirements [9]. The most important areas include electronic packaging [10] as well as coatings for either oxidation [11], corrosion [12] or abrasion protection [13]. Indeed, in all the above-mentioned applications the dissimilar nature of the constituents poses issues in terms of the mechanical integrity and reliability of the whole structure. Failure phenomena of the protective coating include surface cracking, delamination, buckling and spalling [14-16]. All these damage mechanisms arise from the different material properties and from the residual stresses developed during processing within the coating, as well as at the coating/substrate interface. In a previous article, we proposed and studied a novel synthetic strategy for functionally graded polymer nanocomposites based on the motion of magnetic core-shell nanoparticles $\left(\mathrm{Fe}_{3} \mathrm{O}_{4} @ \mathrm{SiO}_{2}\right)$ in a photocurable resin under the application of an external magnetic field gradient, prior to curing [17]. These graded nanocomposites showed to be able to reduce the interfacial stresses resulting from thermal loads [8]. In this work, the residual stresses arising during the photopolymerization process of these graded nanocomposites are evaluated for different gradient morphologies as well as for their homogeneous counterparts. Attention was paid to the influence of the local concentration of the magnetic particles on the decreased photoconversion state of the polymer owing to the light absorption of the particles, and resulting decreased stiffness of the composite. This is made possible by the experimental analysis of the curing kinetics and of the mechanical properties build-up during the photocuring process, and by adapting a Finite Element coupling model [18], usually employed for thermoset resins, to the chemistry and the mechanics of our photocurable material. As a result, it is shown how properly designed functionally graded polymer nanocomposites can reduce by up to $\approx 40 \%$ the interfacial stresses between the polymeric structure and the substrate, therefore minimizing delamination-related problems already at the manufacturing stage.

\section{EXPERIMENTAL SECTION}

\subsection{Materials}

Iron (III) acetyacetonate $(\mathrm{Fe}(\mathrm{acac}) 3,99+\%)$ and benzyl ether $(99 \%)$ were purchased from Acros. Oleylamine (Tech.70\%), polyoxyethylene(5)nonylphenyl ether (Igepal CO-520), ethanol $(\geq 99.5 \%)$ and the epoxy embedding medium kit were purchased from Aldrich. 
Cyclohexane (p.A.) was purchased from Applichem. Ammonia solution $(25 \% \mathrm{~min})$ and tetraethyl orthosilicate (TEOS, $\geq 99 \%$ ) were purchased from VWR and Merck, respectively. The hyperbranched polyester acrylated oligomer (HBP, commercial name CN2302) was purchased from Sartomer, whereas the photo-initiator (Lucirin TPO) was purchased from BASF.

\subsection{Synthesis of $\mathrm{Fe}_{3} \mathrm{O}_{4} @ \mathrm{SiO}_{2}$ nanoparticles}

The synthetic procedure for $\mathrm{Fe}_{3} \mathrm{O}_{4} @ \mathrm{SiO}_{2}$ nanoparticles has been reported in previous publications [17,19]. Briefly, $\mathrm{Fe}_{3} \mathrm{O}_{4} \mathrm{NPs}$ were synthesized dissolving $\mathrm{Fe}(\mathrm{acac})_{3}$ in a mixture of benzyl ether and oleylamine. The solution was heated to $260^{\circ} \mathrm{C}$ and aged at this temperature for $1 \mathrm{~h}$. After that, ethanol was added to precipitate the nanocrystals, which were then separated by centrifugation and dispersed in cyclohexane. In a typical synthesis of $\mathrm{Fe}_{3} \mathrm{O}_{4} @ \mathrm{SiO}_{2} \mathrm{NPs}$, the dispersion of oleylamine-coated $\mathrm{Fe}_{3} \mathrm{O}_{4} \mathrm{NPs}$ in cyclohexane was added to cyclohexane and mixed with Igepal CO-520. The $\mathrm{pH}$ was adjusted to 10 through the addition of ammonium hydroxide and the formation of a transparent brown microemulsion was ensured with the aid of a sonicator. Then, TEOS was added and the reaction was stirred at room temperature for $48 \mathrm{~h}$.

\subsection{Preparation of nanocomposites}

Photoinitiator was dissolved in the HBP in the desired proportion at $65^{\circ} \mathrm{C}$ and stirred for 15 minutes. Nanocomposites containing core-shell nanoparticles were prepared mixing a certain amount of dried $\mathrm{Fe}_{3} \mathrm{O}_{4} @ \mathrm{SiO}_{2} \mathrm{NPs}$ in the HBP and promoting their dispersion with the addition of a small amount of hexane. The mixture was then stirred for one hour at room temperature and sonicated for another hour. The solvent was hence evaporated under vacuum until no more weight variation of the suspension was detected. The effective nanoparticles content of the different formulations was calculated starting from the weight residual as from thermal analyses and converting it to particle volume \% through the densities of the constituents [19]. The studied nanocomposites contained either 4 or $12 \mathrm{vol} \%$ of nanoparticles.

\subsection{Methods}

The heat of the photopolymerization reactions was measured by means of differential scanning calorimetry (DSC Q100, TA Instruments), modified with a photo-DSC set up. A UV lamp with a $200 \mathrm{~W}$ mercury bulb (OmniCure, 2000, Exfo, Canada) was connected to the DSC cell by means of an optical fiber. The cell was sealed with a quartz window that let the UV light pass onto the open aluminum sample pans. The sample space was flushed with nitrogen and the reaction was considered completed when it was no longer possible to detect a change in heat flux. The heat of polymerization was recorded as a function of time and the double bond conversion $x$ was calculated according to Hoyle [20] from the total heat of reaction calculated by integrating the exotherm:

$$
x=\frac{H_{H B P}}{H_{100 \%}}=\frac{H_{\text {total }} / m_{H B P}}{[A G] \cdot \Delta H_{A G}}
$$

where $H_{H B P}$ is the heat of reaction per gram of HBP, $H_{\text {total }}$ the measured heat of reaction per gram of sample, $H_{100 \%}$ the theoretical heat for $100 \%$ double bond conversion of the HBP, $m_{H B P}$ the weight fraction of HBP, $[A G]$ the concentration of acrylate groups in the HBP, and $\Delta H_{A G}$ is the energy of the acrylate double bond equal to $86.31 \mathrm{~kJ} \cdot \mathrm{mol}^{-1}[21]$. 
Dynamic mechanical analysis (DMA Q800, TA Instruments) of HBP at different final conversions was performed at $0.1 \mathrm{~Hz}$, at a constant strain of $0.5 \%$ and at room temperature. Samples $\sim 3 \mathrm{~mm}$ wide and $\sim 0.2 \mathrm{~mm}$ thick were obtained by photopolymerization under nitrogen with the same OmniCure $2000 \mathrm{UV}$ lamp used for the photoDSC experiments. A constant final thickness was ensured by carrying out the polymerization between two quartz plates maintained at a relative distance of $\sim 0.2 \mathrm{~mm}$ by polymer spacers.

Polymerization shrinkage during photopolymerization was monitored real-time using a Michelson interferometer, following the method proposed by de Boer [22]. A detailed description of the measurement method and of the set-up employed is reported elsewhere [23]. A $100 \mu \mathrm{m}$ thick layer of sample was spread on a laboratory glass using a doctor blade. The reaction chamber was flushed with nitrogen and the onset of illumination was synchronized with the data acquisition by sending an electrical signal to the acquisition system while switching on the UV lamp. A typical sampling frequency was $200 \mathrm{~Hz}$. The linear shrinkage $S_{L}$ was calculated according to the following equations:

$$
\begin{aligned}
S_{L} & =\frac{N_{M M} \Delta h_{c}}{h_{c, f i}+N_{M M} \Delta h_{c}} \\
\Delta h_{c} & =\frac{\lambda}{4 n_{c}(t)}=\frac{635 \mathrm{~nm}}{4 n_{c}}
\end{aligned}
$$

where $N_{M M}$ is the number of maxima and minima of the interference signal, $\Delta h_{c}$ is the thickness change between a maximum and a minimum, $h_{c, f i}$ is the final thickness of the coating, $\lambda$ is the wavelength of the laser, $n_{c}$ is the refractive index of the coating, and $t$ is the time. The refractive index was given by the supplier $\left(n_{c}=1.473\right)$ and the final thickness of the sample was measured with a digital caliper (Pro-Max, Fowler). Light intensities were always checked with a Solatell SolaCheck ${ }^{\circledR}$ UV spectrometer.

Curing degrees of the samples prepared for DMA, interferometer and DSC tests were compared between each other by means of Fourier Transform Infrared Analysis, which were acquired using a Perkin Elmer Spectrum One MIR $\left(600-4000 \mathrm{~cm}^{-1}\right)$ with an ATR accessory. The spectra were acquired with 32 scans and a resolution of $4 \mathrm{~cm}^{-1}$.

\section{EXPERIMENTAL CHARACTERIZATION}

\subsection{Mechanical properties and chemical shrinkage}

In order to be able to correlate the chemistry and the mechanics of the photopolymerization process with the development of the residual stresses, the reaction kinetics and the evolution of the modulus and of the shrinkage during the whole reaction was taken into account. While the kinetics were studied by means of photo-DSC, elastic modulus and shrinkage changes were evaluated through DMA and interferometry analyses, respectively. However, the direct correlation between the different measurements was hindered by the fast evolution of the photocuring reaction and the relatively low temporal resolution of the employed techniques. This issue was overcome through a discrete approach, in which the hyperbranched polymer was characterized at different steady-state curing degrees.

Four different HBP set of samples were prepared by adjusting the amount of photoinitiator they contained and the UV-light intensity to which they were exposed, as indicated in Table 
1. For these sample types, photo-DSC and interferometry tests were carried out and DMA samples were prepared with the same processing conditions. Additionally, the final conversion of each sample was assessed through FTIR analyses, by monitoring the intensity of the $\mathrm{C}=\mathrm{C}$ double bond peak centered at $1610 \mathrm{~cm}^{-1}$, normalized with the intensity of the carbonyl peak centered at $1760 \mathrm{~cm}^{-1}$.

Table 1. Photoinitiator (Lucirin TPO) concentration and UV-light intensity utilized for the kinetics study of the four different formulations of the pure HBP. The average steady-state final conversions, their standard deviations and the conversion curves are also reported.

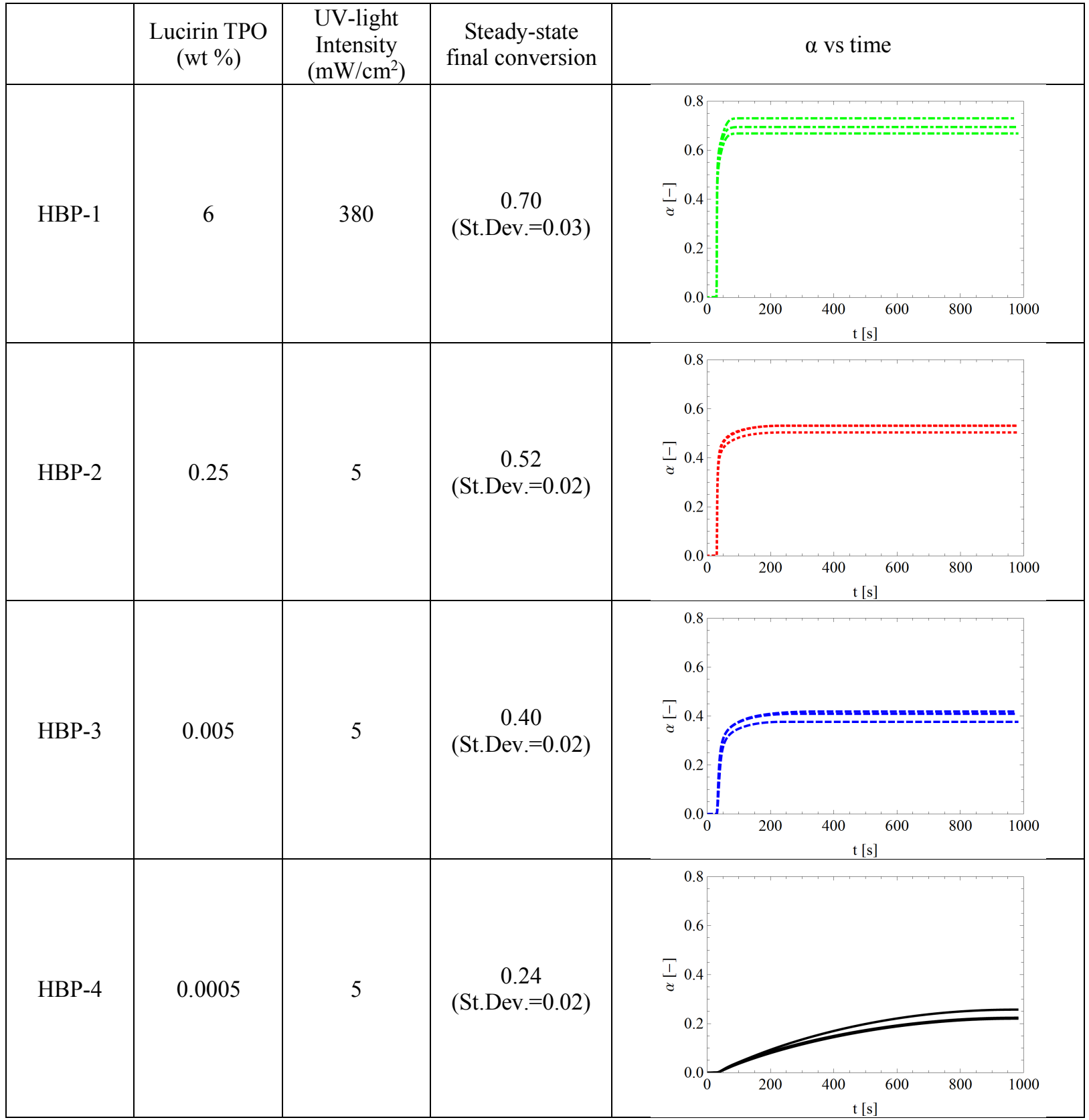

The four different studied final conversion stages were achieved by employing different combinations of photoinitiator contents and UV-light irradiation intensities (Table 1). The whole range of final conversions from the unpolymerized material to the fully cured one was covered, ensuring a complete characterization at every degree of conversion. HBP-1 contained the same amount of photoinitiator of the graded nanocomposite formulations 
$(6 \mathrm{wt} \%)$ and, similarly to them, its curing reaction was performed at $380 \mathrm{~mW} / \mathrm{cm}^{2}$ (nanocomposites were irradiated from both sides using two UV lamps, each of which ensured an irradiation intensity of $190 \mathrm{~mW} / \mathrm{cm}^{2}$ [17]). The three other formulations (HBP-2, HBP-3 and HBP-4) contained a lower amount of photoinitiator and were irradiated with a $5 \mathrm{~mW} / \mathrm{cm}^{2}$ UV-light, which ensured a steady-state final conversion in the desired range. From DSC analyses, the final steady-state conversion of the four formulations were $0.70,0.52,0.40$ and 0.24 . The storage and loss moduli obtained in the DMA tests at $0.1 \mathrm{~Hz}$ are reported in Figure 1 for the four steady-state relative conversions, defined as:

$$
\alpha_{r}=\frac{\alpha}{\alpha_{\max }}
$$

Where $\alpha$ is the conversion at any given time and $\alpha_{\max }$ is the maximum conversion.

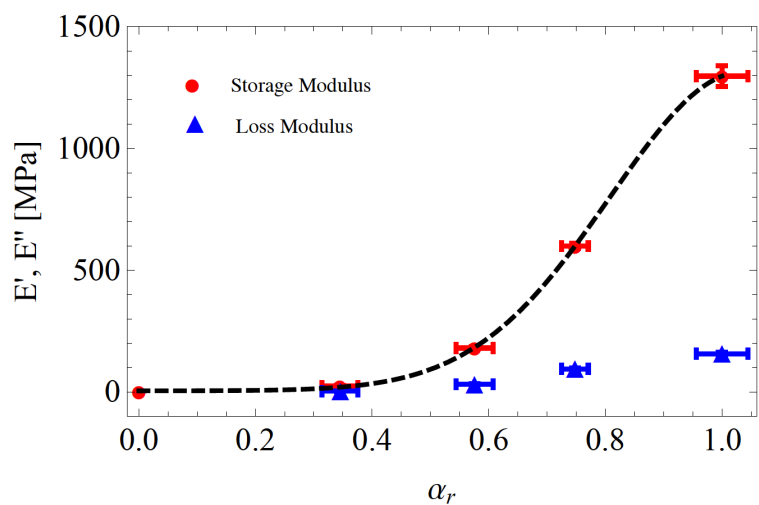

Figure 1. Evolution of the storage modulus, $E^{\prime}$, and loss modulus, $E^{\prime \prime}$, of the hyperbranched resin as a function of its relative conversion. Experimental data were fitted by a sigmoidal function.

In all cases, the loss modulus was around one order of magnitude smaller than the storage one, leading us to neglect viscoelastic stress relaxation. The evolution of the experimental $E$, as a function of the relative conversion $\alpha_{r}$ was accurately fitted with a sigmoidal curve, which was expressed as:

$$
E^{\prime}=d+e^{a+b x+c x^{2}}
$$

where the parameters $a, b, c$ and $d$ were found to be $-2.83,19.23,-9.24$ and $4.24 \mathrm{MPa}$, respectively. It is worthy to notice that the storage modulus did not increase until $\alpha_{r}$ reached a value $\approx 0.4$, which approximates the conversion at which gel point occurs and the material can efficiently transfer load. $E$ ' continues to increase up to a value of $\sim 1.3 \mathrm{GPa}$, obtained at full relative conversion.

The photocuring shrinkage experimentally measured with the interferometer is reported in Figure 2 for the four steady-state relative conversions. The linear shrinkage linearly increased with conversion (slope equal to 3.60). This result is in agreement with what already found for linear polymers [22] and confirms that the shrinkage is proportional to the fraction of reacted chemical bonds, at least up to the vitrification point $[22,24]$. 


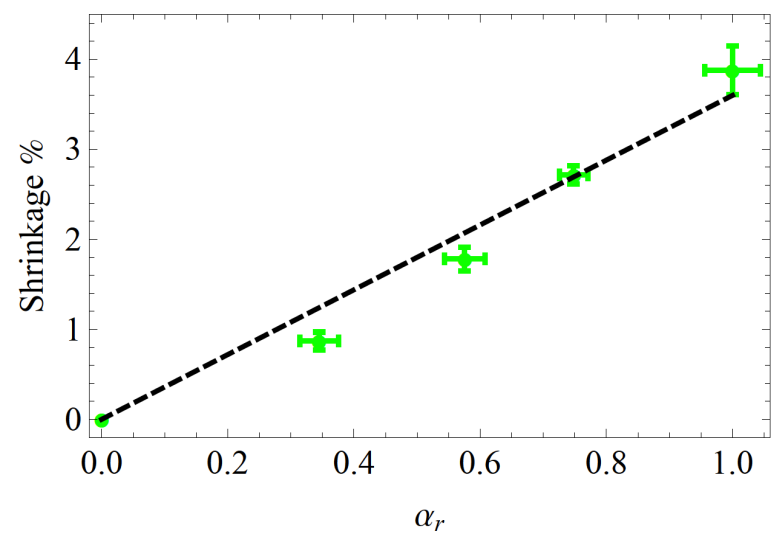

Figure 2. Evolution of the curing shrinkage of the hyperbranched resin as a function of its relative conversion. Experimental data with the corresponding standard deviations are shown in green and fitted by a linear function.

\subsection{Characterization of the photopolymerization kinetics}

The cure kinetics of the pure $\mathrm{HBP}$ and of the nanocomposites (i.e. $\mathrm{HBP}+\mathrm{Fe}_{3} \mathrm{O}_{4} @ \mathrm{SiO}_{2} \mathrm{NPs}$ ) was modeled assuming an autocatalytic behavior [25,26] (Figure 3). In addition, being opaque, magnetic nanoparticles influence the photopolymerization process in a way that is directly related to their concentration $\phi$ [19]. The modified autocatalytic model employed to reproduce the experimental measurements can be expressed as:

$$
\frac{d \alpha}{d t}=k \alpha_{r}^{m}\left(1-\alpha_{r}\right)^{n}
$$

where $\alpha$ is the conversion degree, $\alpha_{r}$ is the relative conversion as defined in equation $4, m$ is the autocatalytic exponent, $n$ is the reaction order, and $k$ is a function of the volume fraction $\phi$ and represents the reaction rate. The autocatalytic exponent takes into account the autoacceleration of the photopolymerization reaction caused by the immobilization of the polymer chains in the forming network due to an increase in viscosity that prevents recombination of the polymer radicals, decreasing the termination rate.
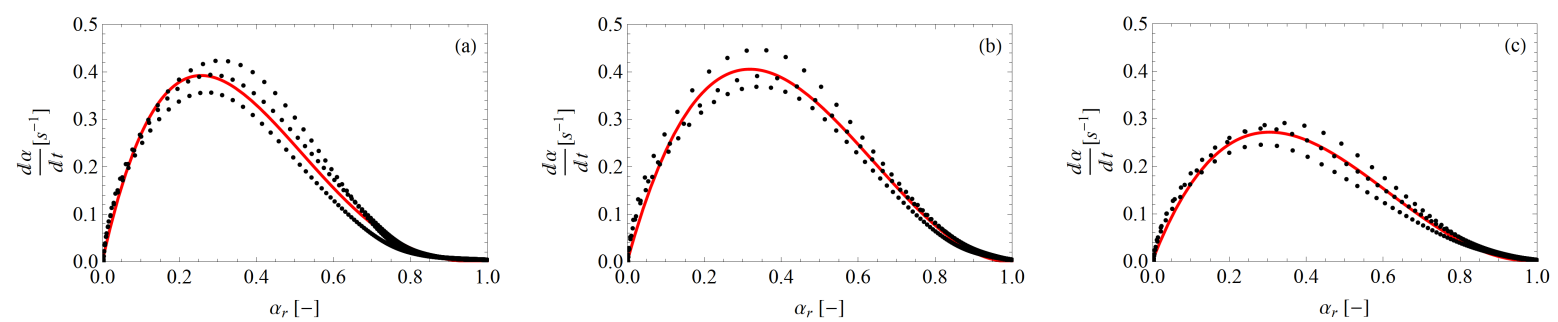

Figure 3. Fitting of the experimental curves for the pure HBP (a) and for the nanocomposites con-taining 4 (b) and 12 (c) vol\% of $\mathrm{Fe}_{3} \mathrm{O}_{4} @ \mathrm{SiO}_{2}$ nanoparticles. The maximum conversion was defined as the conversion for which the polymerization rate was smaller than $1 \%$ of the maximum polymerization rate [24].

The rate constant $k$ was fitted with an exponentially decaying function assuming that it becomes negligible as $\phi$ approaches 1 (Figure 4). The autocatalytic exponent $m$ and the reaction order exponent $n$ were assumed to be constant instead, and taken equal to the average of the values $m(\phi)$ and $n(\phi)$. 

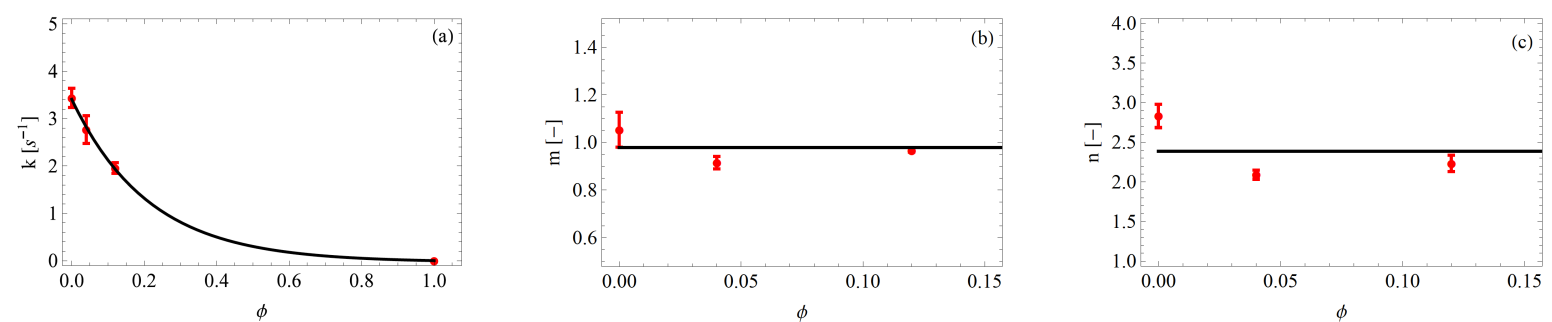

Figure 4. Plots of the rate constant $k(a)$, of the autocatalytic exponent $m$ (b) and of the reaction order exponent $n$ (c) as a function of the $\mathrm{Fe}_{3} \mathrm{O}_{4} @ \mathrm{SiO}_{2} \mathrm{NPs}$ volume fraction $\phi$.

\section{NUMERICAL MODEL}

The equations describing the reaction kinetics, the chemical shrinkage and the evolution of the mechanical properties during curing were implemented in a user material subroutine (UMAT) in the commercial finite element software (Abaqus 11.0), following the scheme proposed by Rabearison et al. [18]. The curing degrees were initially calculated using a forward Euler integration of eq. 5 in which the rate of curing was evaluated in the actual step $(t)$ to determine the degree and the rate of curing for the following step $(t+1)$ (Figure 5). Although not predicting the small inflexions of the conversion curves arising at increasing particle volume fractions (Figure $5 \mathrm{c}$ ), the integration was able to accurately match the final conversions of all the studied formulations.
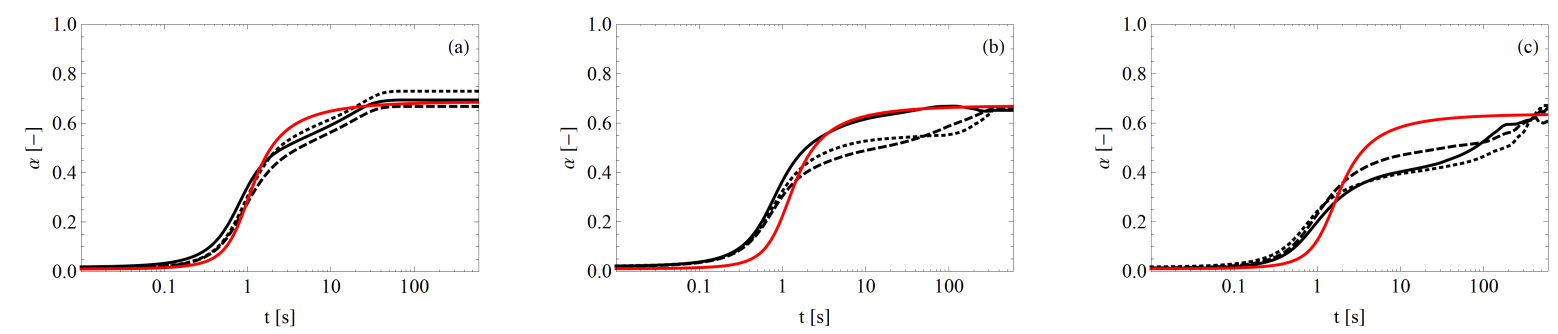

Figure 5. Conversion curves as from experiments (black) and after integration of Equation 4 (red) for the pure $\mathrm{HBP}(\mathrm{a})$ and the nanocomposites filled with 4 (b) and 12 (c)vol\% of Fe3 $\mathrm{O}_{4} @ \mathrm{SiO}_{2}$ nanoparticles.

Once the degree of curing was obtained, the elastic modulus and the coefficient of chemical shrinkage of the photocurable matrix were directly computed from the empirical equations derived in the section "Mechanical properties and chemical shrinkage". Furthermore, knowing the volumetric fraction and the elastic properties of the reinforcement $\mathrm{Fe}_{3} \mathrm{O}_{4} @ \mathrm{SiO}_{2}$ nanoparticles, the evolution of the mechanical properties of the photocurable nano-composite was determined using a Mori Tanaka's micro-mechanics model [27-29]. In this homogenization method, the stiffness tensor of the composite material ( $\left.\mathbf{L}_{\mathrm{comp}}\right)$ is given by:

$$
\mathbf{L}_{\text {comp }}=\mathbf{L}_{m}+v_{p} \cdot\left(\mathbf{L}_{p}-\mathbf{L}_{m}\right) \cdot \mathbf{A}_{m t}
$$

where $\mathbf{L}_{m}$ and $\mathbf{L}_{p}$ are the stiffness tensors of the matrix and the nano-particles, respectively. $\mathbf{v}_{\mathrm{p}}$ is the volumetric fraction of the nano-particles and $\mathbf{A}_{\mathrm{mt}}$ is the strain concentration tensor:

$$
\mathbf{A}_{m t}=\mathbf{A}_{d i l}\left[\left(1-v_{p}\right) \cdot \mathbf{I}+v_{p} \cdot \mathbf{A}_{d i l}\right]^{-1}
$$

$\mathbf{A}_{\text {dil }}$ being the dilute strain concentration tensor:

$$
\mathbf{A}_{d i l}=\left[\mathbf{I}+\mathbf{S} \cdot \mathbf{L}_{m}^{-1}\left(\mathbf{L}_{i}-\mathbf{L}_{m}\right)\right]^{-1}
$$


and $\mathbf{S}$ is the Eshelby tensor. The chemical shrinkage of the composite could be determined from a homogenization model as:

$$
\boldsymbol{\beta}_{\text {comp }}=\boldsymbol{\beta}_{m}+v_{p} \cdot\left\{\left(\mathbf{L}_{i}-\mathbf{L}_{m}\right) \cdot\left[\mathbf{S}-v_{p} \cdot(\mathbf{S}-\mathbf{I})\right]\right\}^{-1} \cdot \mathbf{L}_{p}\left(\boldsymbol{\beta}_{p}-\boldsymbol{\beta}_{m}\right)
$$

Where $\boldsymbol{\beta}_{\mathrm{comp}}, \boldsymbol{\beta}_{\mathrm{p}}$ and $\boldsymbol{\beta}_{\mathrm{m}}$ are the chemical shrinkage tensor of the composite, the particles and the matrix. [30]

The geometrical model employed to study the residual strains in functionally graded nanocomposites consisted in a column of $150 \times 1 \times 1 \mu \mathrm{m}^{3}$ representing a coating of $150 \mu \mathrm{m}$ thick over a $300 \mu \mathrm{m}$ thick substrate (Figure 6). For sake of simplicity, perfect bonding was assumed between the coating and the substrate. Symmetric boundary conditions were applied along the length of the column to represent an infinite medium.

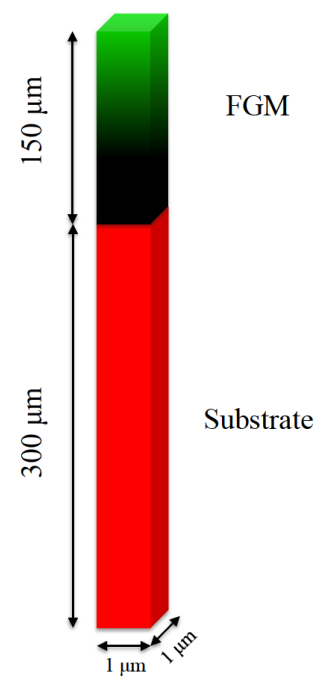

Figure 6. Representation of the coating-substrate structure as implemented in the FEM model. Dimensions do not show at proper scale.

Nine different nanoparticle distributions along the thickness of the coating, based on three different concentrations $(8,12,15 \mathrm{vol} \%)$ and three different morphologies, were simulated to study the interfacial residual stresses arising from the photopolymerization process. The different morphologies comprised homogeneous distributions of nanoparticles as well as linear and "S-shaped" graded distributions obtained through the application of a magnetic field (Figure 7).
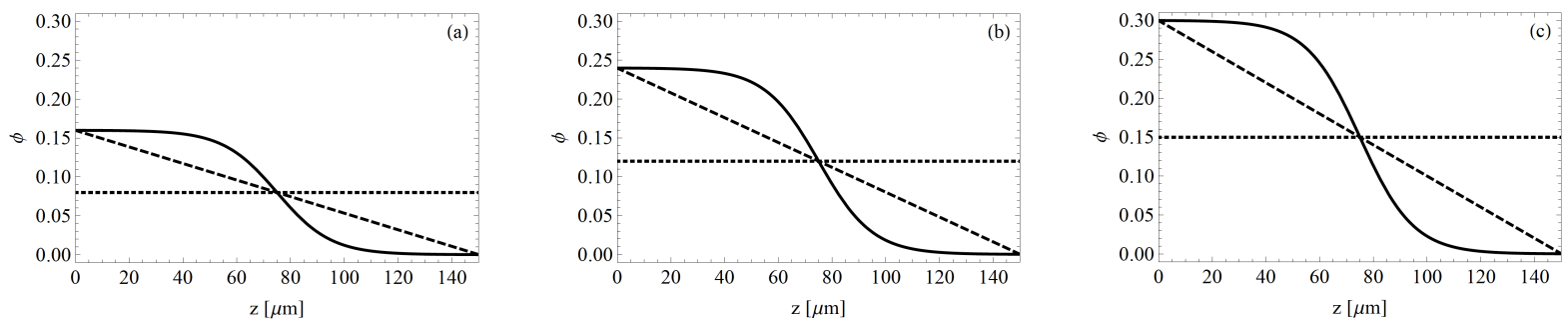

Figure 7. Plots of the simulated graded and homogeneous structures. Solid lines represent S-shape gradients, whereas dashed and dotted lines represent linear gradients and homogeneous distributions, respectively. 


\section{PHOTOPOLYMERIZATION ANALYSIS}

Figure 8 illustrates the variation of the degree of curing of the photo-polymerized matrix for different volumetric fraction of nanoparticles. The maximum degree of curing achieved after UV exposing during $600 \mathrm{~s}$ was $69 \%$ and $56 \%$ for volumetric reinforcement fractions of 0 and $30 \%$, respectively.

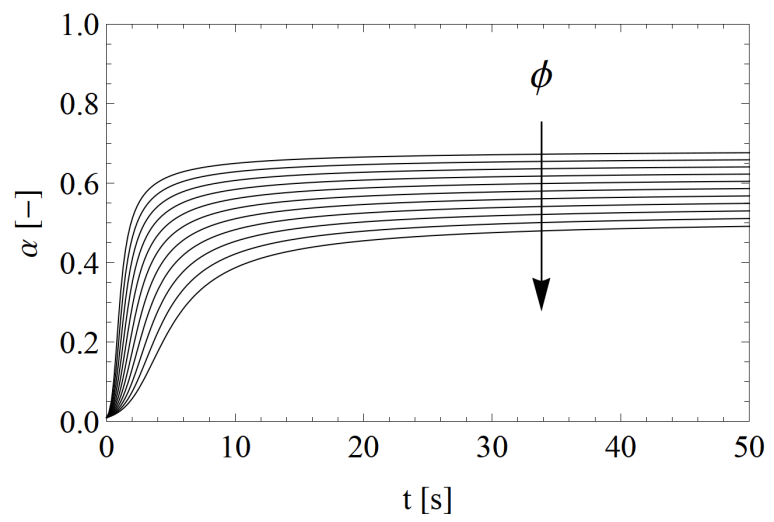

Figure 8. Variation of the conversion ( $\alpha$ ) with respect to the time for volumetric fractions of particles ranging from 0 (top) to $30 \%$ (bottom), with 3 vol\% increments. To highlight the behavior at the beginning of the reaction, only the first 50 seconds are shown.

On one hand, the incorporation of nanoparticles in the polymeric matrix reduced its chemical shrinkage (Figure 9a) and elastic modulus (Figure 9b, red line) due to a concurrent decrease in its final conversion. On the other hand, the presence of the hard nanoparticles increased the elastic properties of the composite material (Figure 9b, black line). These two competing effects resulted in an overall stiffening of the whole structure, which showed a maximum in the elastic properties for a volumetric reinforcement fraction of $\approx 23 \%$ (Figure $9 \mathrm{~b}$, black line).
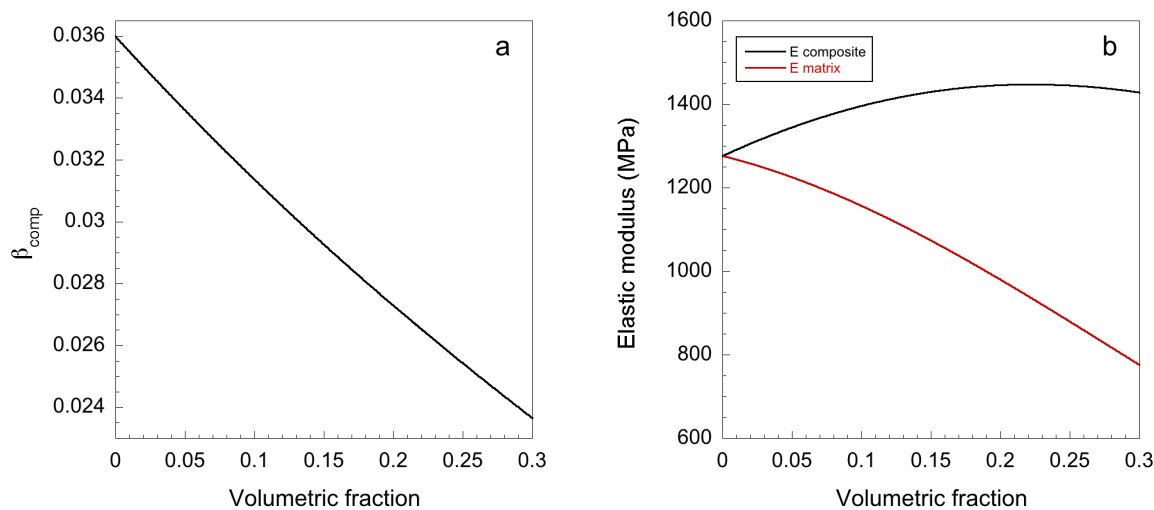

Figure 9. Evolution of a) the composite shrinkage coefficient $\left(\beta_{\text {comp }}\right)$ and b) matrix and composite elastic modulus ( $E_{\text {mat }}$ and $E_{\text {comp }}$ ) respect to the volumetric fraction of nanoparticles.

\section{NUMERICAL RESULTS}

The profiles of the stress in the plane of the coating/substrate interface through the coating and the substrate $\left(E_{\text {substrate }}=10 \mathrm{GPa}\right)$ are plotted in Figure 10 for different distributions of nanoparticles. The simulations of the homogeneous nanocomposites (with volumetric reinforcement fractions of $0,8,12$ and $15 \%$ ) showed that the residual stresses obtained after the photopolymerization are inversely proportional to the nanoparticle content. Thus, the higher the volume fraction, the smaller the residual stresses in the coating $(\approx 20,18,16.8$ and 
$16 \mathrm{MPa}$ for the increasing homogeneous concentration of nanoparticles). The simulations of the non-homogeneous nanoparticles distribution showed that the graded composites are able to locally reduce the residual stresses (Figure $9 \mathrm{~A}$ to $\mathrm{C}$ ). Therefore, a higher concentration of particles close to the substrate leads to a local reduction of the residual stresses at the coating/substrate interface. It is worthy noticing that the maximum residual stresses in the simulations could be overestimated due to the assumptions of a perfect interface and a pure elastic behavior of the polymer during curing. Nonetheless, it can be reasonably assumed that the mechanism of interfacial stress reduction seen in the simulations for the graded composites would arise in the real setting. Indeed, going towards the free surface, stresses increase in a way that is inversely proportional to the nanoparticles concentration and independent of the shape of the distribution (from $\approx 12 \mathrm{MPa}$ at the interface to $\approx 20 \mathrm{MPa}$ at the free surface for a variation in nanoparticles concentration ranging from $30 \%$ at the interface to $0 \%$ at the surface). An equivalent response was obtained from the simulations of the inverse distribution of nanoparticles (Figure $9 \mathrm{D}$ to F). When the reinforcement was concentrated at the free surface, a reduction of the stresses in the surface vicinity was produced. On the other hand, the particle-depleted coating/substrate interface experienced residual stresses as high as those generated in the pure polymer.
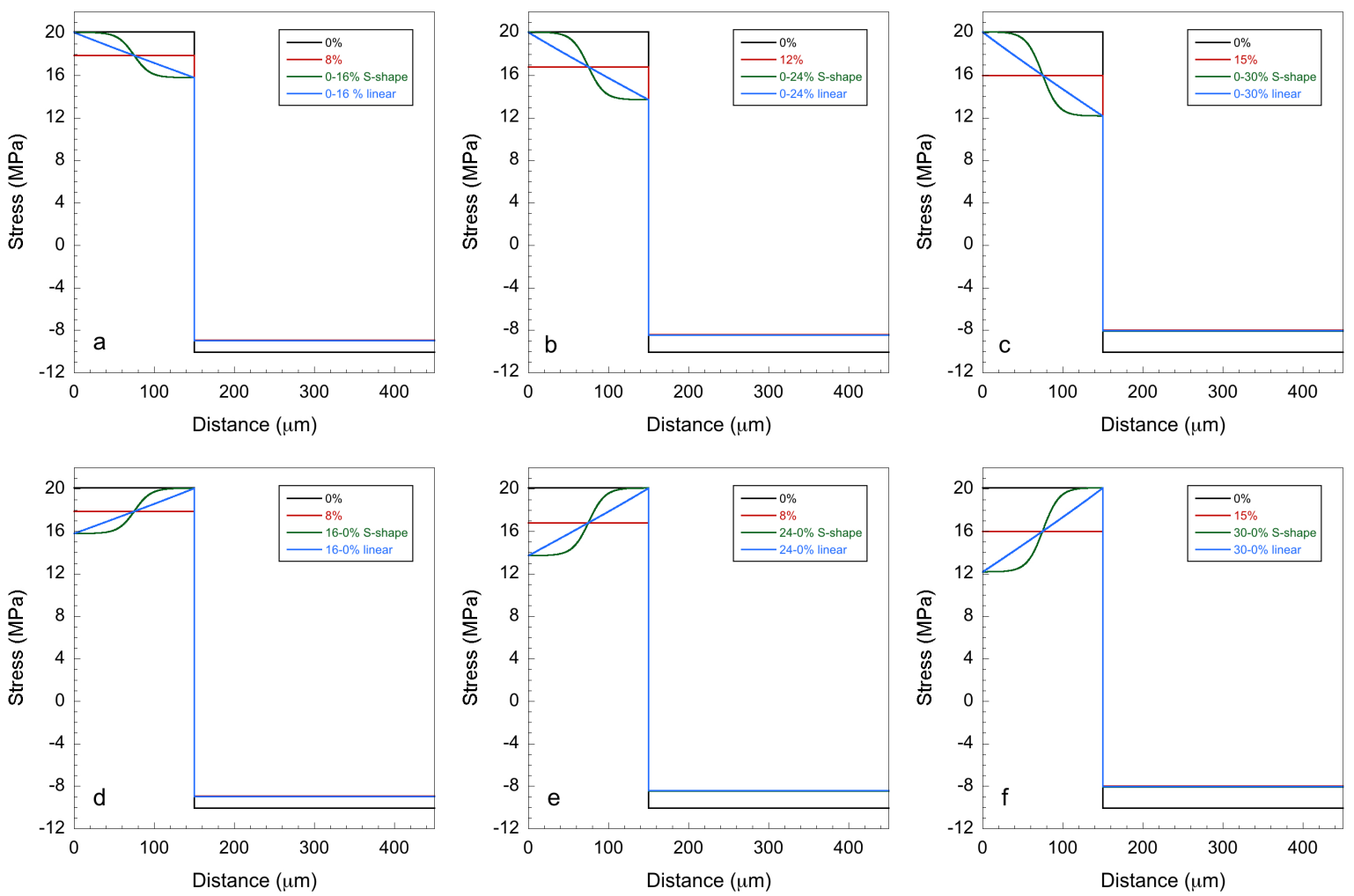

Figure 10. Profile of the stress in the substrate plane for the homogeneous, linear and "S-shaped" nanoparticle distributions for different volumetric fractions: A) 0-16\%, B) 0-24\%, C) 0-30\% and their corresponding inverse distributions ( $D$ to $F$ ).

Although useful, the experimental validation of the obtained numerical results does not appear to be straightforward. Indeed, the classical beam bending technique usually employed to measure stresses arising in coatings would be insensitive to changes in the distribution of the nanoparticles. This is due to the fact that differences between graded and homogeneous composites would only come from a shift in the position of the neutral axis, which would be small compared with the total thickness of the coating/substrate system, while the average stress would be unchanged. 


\section{PARAMETRIC STUDY}

Three parametric studies were carried out, starting with looking at the effect of substrate stiffness on the magnitude of the resulting stresses. As observed in Figure 11, the soft substrate $(1 \mathrm{GPa})$ gave rise to lower residual stress compared to the stiffer one $(100 \mathrm{GPa})$. As a matter of fact, stresses at the coating/substrate interface deriving from the chemical shrinkage of the coating can be efficiently reduced through the selection of the most appropriate substrate.

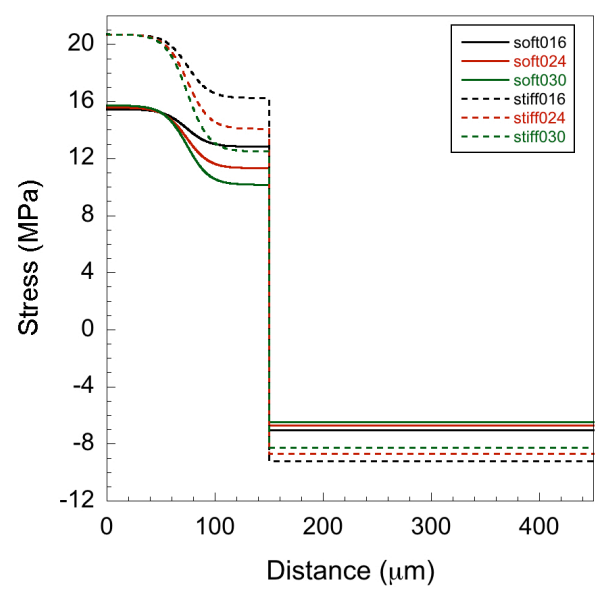

Figure 11. Three different "S" shape volumetric distribution on two different substrates, soft (1 GPa) and stiff (100 GPa).

The second and third parametric studies focused on the behavior of a composite coating exhibiting a linear nanoparticle gradient ranging from $0 \mathrm{vol} \%$ (at the free surface) to $24 \mathrm{vol} \%$ (at the coating-substrate interface). The aim of the second study was to verify the importance of the dependence of the conversion of the polymeric matrix on the local nanoparticle volume fraction. To do so, the effect of having a matrix with a constant final conversion $\left(\alpha_{\text {constant }}=0.70\right)$ was compared with the results generated by a matrix exhibiting a final conversion dependent on the local nanoparticle volume fraction. In other words, this study compares the effect of a thermally curable matrix versus a photocurable one. Figure 12a indicates that when the matrix conversion is independent of the local particle volume fraction, the stiffening effect generated by the nanoparticles is not outweighed by the weakening caused by a corresponding lower conversion of the matrix, resulting in higher stress concentration at the coating-substrate interface. Therefore, the definition of a particledependent conversion not only reflects the real behavior of the polymer during the photopolymerization process, as explained earlier, but results to be fundamental in view of stress-reducing applications.

The aim of the third study was to check the influence of the stiffness of the reinforcing particles on the stresses generated at the coating-substrate interface. Figure $12 \mathrm{~b}$ shows that, an increment of the stiffness of the particles gives rise to a corresponding increase of the interfacial stresses, as a result of the higher elastic modulus of the composite in the same region. As a result, the stress-reducing effect generated by graded nanocomposites can thus be maximized by the use of compliant nanoparticles, whose stiffening effect can be largely offset by a concurrent decrease in the matrix conversion and modulus. 

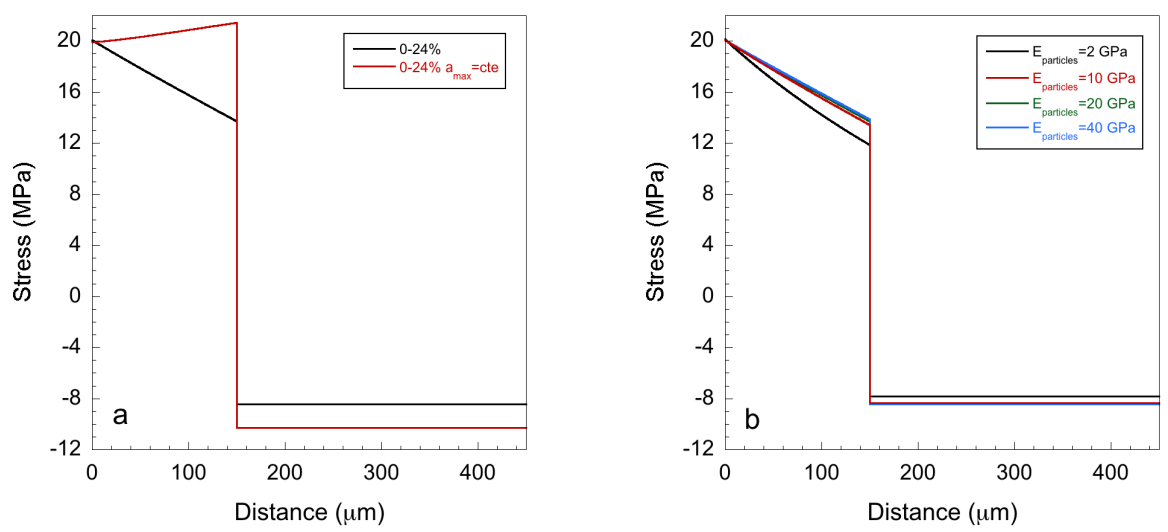

Figure 12. Stress profiles of the composite materials with a linear graded distribution ranging from 0 vol\% (at the free surface) to 24 vol\% (at the coating-substrate interface) (a) in the case of a matrix with a constant (red) and with a particle-dependent (black) conversion, (b) in the case of a particle-dependent conversion of the matrix and different stiffness of the reinforcing particles.

\section{CONCLUSIONS}

For the first time, a thorough experimental analysis of the curing kinetics as well as of the modulus and shrinkage build-up of a photocurable magnetic nanocomposite was coupled with a complementary numerical study of the stress state in the final coating. With this approach, the residual stresses arising during the photopolymerization process of the nanocomposites were evaluated for different gradient and homogeneous morphologies. For the studied materials, it was shown that functionally graded polymer nanocomposites can reduce by up to $\approx 40 \%$ the interfacial stresses between the coating and the substrate compared to the pure polymer, and by up to $\approx 25 \%$ compared to their homogeneous counterparts. The beneficial effect deriving from the concentration of stiff particles at the substrate/coating interface can be further enhanced by the selection of the most appropriate substrate and reinforcing particles, minimizing the detrimental consequences deriving from the chemical shrinkage of the coating. Moreover, it was clearly pointed out how the definition of a particle-dependent conversion of the polymeric matrix, typical of photocurable polymers, resulted to be crucial not only to exhaustively reflect the real behavior of the nanocomposite, but also in order to give rise to consistent stress-reduction effects. All the presented strategies constitute an innovative and effective way to prevent classical problems, such as delamination, encountered in coating technologies as well as in standard laminated composites.

\section{ACKNOWLEDGEMENTS}

The authors acknowledge the Swiss National Science Foundation (project 200020_155888) for financial support.

\section{REFERENCES}

[1] G.M. Luz, J.F. Mano, Biomimetic design of materials and biomaterials inspired by the structure of nacre. Phil. Trans. R. Soc. A 367 (2009) 1587-1605.

[2] A.K. Ray, S.K. Das, S. Mondal, P. Ramachandrarao, Microstructural characterization of bamboo. J. Mater. Sci. 39 (2004) 1055-1060.

[3] J.E. Phillips, K.L. Burns, J.M. Le Doux, R.E. Guldberg, A.J. García, Engineering graded tissue interfaces. Proc. Natl. Acad. Sci. 105 (2008) 12170-12175. 
[4] H. Yao, M. Dao, T. Imholt, J. Huang, K. Wheeler, A. Bonilla, S. Suresh, C. Ortiz, Protection mechanisms of the iron-plated armor of a deep-sea hydrothermal vent gastropod. Proc. Natl. Acad. Sci. 107 (2010) 987-992.

[5] V. Birman, L.W. Byrd, Modeling and analysis of functionally graded materials and structures. Appl. Mech. Rev. 60 (2007) 195-216.

[6] L.L. Shaw, Thermal residual stresses in plates and coatings composed of multi-layered and functionally graded materials. Compos. Part B: Eng. 29 (1998) 199-210.

[7] X.C. Zhang, B.S. Xu, H.D. Wang, Y. Jiang, Y.X. Wu, Modeling of thermal residual stresses in multilayer coatings with graded properties and compositions. Thin Solid Films 497 (2006) 223-231.

[8] T. Nardi, Y. Leterrier, J.-A. E. Månson, Bioinspired functionally graded nanocomposites synthesized through magnetophoretic processes for tailored stress reduction. MRS Online Proceedings Library 1685 (2014) 1-7.

[9] A.G. Evans, J.W. Hutchinson, The thermomechanical integrity of thin films and multilayers. Acta metall. mater. 43 (1995) 2507-2530.

[10] Y. Wen, C. Basaran. Thermomechanical Stress Analysis of Multi-Layered Electronic Packaging. J. Electron. Packaging 125 (2003), 134-138.

[11] F. Smeacetto, M. Salvo, M. Ferraris, Oxidation protective multilayer coatings for carbon-carbon composites. Carbon 40 (2002) 583-587.

[12] J.A. Alegría-Ortega, L.M. Ocampo-Carmona, F.A. Suárez-Bustamante, J.J OlayaFlórez, Erosion-corrosion wear of $\mathrm{Cr} / \mathrm{CrN}$ multi-layer coating deposited on AISI-304 stainless steel using the unbalanced magnetron (UBM) sputtering system. Wear 290291 (2012) 149-153.

[13] S.S Qureshi, Z. Zheng, M.I. Sarwar, O. Félix, G. Decher, Nanoprotective layer-by-layer coatings with epoxy components for enhancing abrasion resistance : toward robust multimaterial nanoscale films. ACS Nano 7 (2013) 9336-9344.

[14] X. Zhang, B. Xu, H. Wang, Y. Wu, Thermal residual stresses in multilayered coatings. J. Mater. Sci. Technol. 21 (2005) 599-605.

[15] Y. Leterrier, A. Mottet, N. Bouquet, D. Gilliéron, P. Dumont, A. Pinyol, L. Lalande, J.H. Waller, J.-A. E. Månson, Mechanical integrity of thin inorganic coatings on polymer substrates under quasi-static, thermal and fatigue loadings. Thin Solid Films 519 (2010) 1729-1737.

[16] M. Sebastiani, C. Eberl, E. Bemporad, G.M. Pharr, Depth-resolved residual stress analysis of thin coatings by a new FIB-DIC method. Mat. Sci. Eng. A-Struct. 528 (2011) 7901-7908.

[17] T. Nardi, Y. Leterrier, A. Karimi, J.-A.E. Månson, A novel synthetic strategy for bioinspired functionally graded nanocomposites employing magnetic field gradients. RSC Adv. 4 (2014) 7246-7255.

[18] N. Rabearison, C. Jochum, J.C. Grandidier, A FEM coupling model for properties prediction during the curing of an epoxy matrix. Comp. Mater. Sci. 45 (2009) 715-724.

[19] T. Nardi, M. Sangermano, Y. Leterrier, P. Allia, P. Tiberto, J.-A.E. Månson, UV-cured transparent magnetic polymer nanocomposites. Polymer 54 (2013) 4472-4479.

[20] S.P. Pappas, Radiation curing: science and technology. Plenum Press, New York, 1992.

[21] K.S. Anseth, C.M. Wang, C.N. Bowman, Reaction behaviour and kinetic constants for photopolymerizations of multi(meth)acrylate monomers. Polymer 35 (1994) 3243-3250.

[22] J. de Boer, R.J. Visser, G.P. Melis, Time-resolved determination of volume shrinkage and refractive index change of thin polymer films during photopolymerization. Polymer 33 (1992) 1123-1126.

[23] L.E. Schmidt, Low stress acrylated hyperbranched polymers. Ph.D. Thesis, EPFL, Lausanne, Switzerland, 2006. 
[24] C.N. Bowman, N.A. Peppas, Coupling of kinetics and volume relaxation during polymerizations. Macromolecules 24 (1991) 1914-1920.

[25] M.R. Kamal, Thermoset characterization for moldability analysis. Polym. Eng. Sci. 14 (1974) 231-239.

[26] S. Dalle Vacche, V. Geiser, Y. Leterrier, J.-A.E. Månson, Time-intensity superposition for photoinitiated polymerization of fluorinated and hyperbranched acrylate nanocomposites. Polymer 51 (2010) 334-341.[27] T. Mori, K. Tanaka, Average stress in matrix and average elastic energy of materials with misfitting inclusions. Acta Metall. 21 (1973) 571-574.

[28] Y. Benveniste, A new approach to the application of Mori-Tanaka's theory in composite. Mech. Mater. 6 (1987) 147-157.

[29] J.D. Eshelby, The determination of the elastic field of an ellipsoidal inclusion and related problems. Proc. R. Soc. Lond. A 241 (1957) 376-396.

[30] P. Lu, Further studies on Mori-Tanaka models for thermal expansion coefficients of composites. Polymer 54 (2013) 1691-1699. 